\title{
Blood pressure measurements and hypertension in infants, children, and adolescents: from the postmercury to mobile devices
}

\author{
Seon Hee Lim, MD ${ }^{1}$, Seong Heon Kim, MD² \\ ${ }^{1}$ Department of Pediatrics, Uijeongbu Eulji Medical Center, Uijeongbu, Korea; ${ }^{2}$ Department of Pediatrics, Seoul National University Children's Hospital, Seoul National \\ University College of Medicine, Seoul, Korea
}

A mercury sphygmomanometer (MS) has been the gold standard for pediatric blood pressure (BP) measurements, and diagnosing hypertension is critical. However, because of environmental issues, other alternatives are needed. Noninvasive BP measurement devices are largely divided into auscultatory and oscillometric types. The aneroid sphygmomanometer, the currently used auscultatory method, is inferior to MS in terms of limitations such as validation and regular calibration and difficult to apply to infants, in whom Korotkoff sounds are not audible. The oscillometric method uses an automatic device that eliminates errors caused by human observers and has the advantage of being easy to use; however, owing to its measurement accuracy issues, the development of an international validation protocol for children is important. The hybrid method, which combines the auscultatory and electronic methods, solves some of these problems by eliminating the observer bias of terminal digit preference while maintaining measurement accuracy; however, the auscultatory method remains limited. As the agerelated characteristics of the pediatric group are heterogeneous, it is necessary to reconsider the appropriate BP measurement method suitable for this indication. In addition, the mobile application-based BP measurement market is growing rapidly with the development of smartphone applications. Although more research is still needed on their accuracy, many experts expect that mobile application-based BP measurement will effectively reduce medical costs due to increased ease of access and early BP management.

Key words: Blood pressure, Child, Mercury, Oscillometry, Auscultatory

\section{Key message}

- Hypertension is defined as a blood pressure (BP) $>90$ th (elevated) or 95th (hypertension) percentile in children by height, age, and sex and $>95$ th percentile in neonates by age, birth weight, and sex.

- Although the oscillometric method can be used for screenings, the auscultatory method remains the gold standard. The hybrid method employs the auscultatory and electronic methods and can reduce bias.

- BP measurement mobile device applications have a potential for development.

\section{Introduction}

Blood pressure (BP) measurement is a common and imperative component of physical examinations in children and adults. Although the prevalence of hypertension in children is lower than that in adults, its early detection is crucial for preventing later complications. Before recognizing the $\mathrm{BP}$ as normal or high, we must ensure its accurate measurement. BP in children can be affected by several factors such as measurement technique, emotional state, and activity level. The accommodation effect and measurement device type are 2 critical factors for BP measurement accuracy in children. The accommodation effect refers to the fact that $\mathrm{BP}$ usually decreases as measurements are repeated. Therefore, multiple BP measurements are recommended before the diagnosis of elevated $\mathrm{BP}^{1)}$ There are several methods for measuring BP, including the mercury sphygmomanometer (MS). First introduced more than 110 years ago, the MS has been the gold standard method for obtaining BP and diagnosing hypertension in children. However, because of environmental issues related to mercury toxicity, the MS will no longer be used. This review article describes several BP measurement methods and speculates on what may replace MS in children.

\footnotetext{
Corresponding author: Seong Heon Kim, MD. Department of Pediatrics, Seoul National University Children's Hospital, Seoul National University College of Medicine, 101 Daehak-ro, Jongno-Gu, Seoul 03080, Korea 


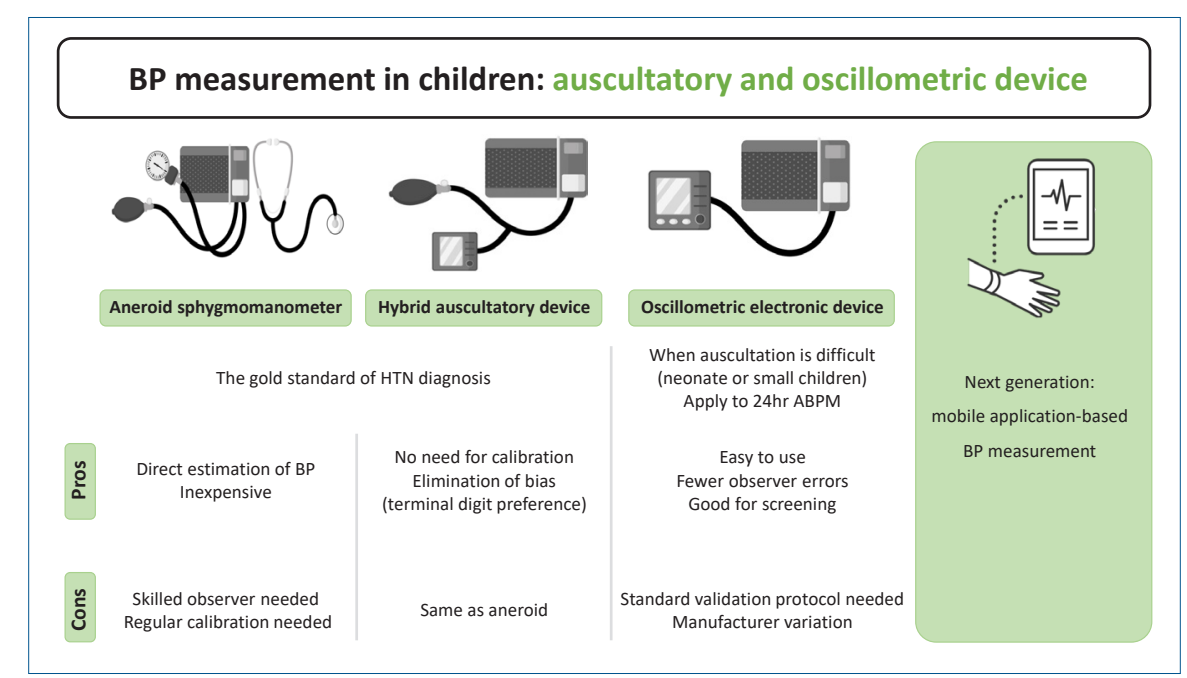

Graphical abstract. BP, blood pressure; HTN, hypertension; ABPM, ambulatory BP monitoring.

\section{Definition of hypertension in children}

\section{Definition of pediatric hypertension}

After the American Heart Association (AHA) and American College of Cardiology proposed that a BP higher than 115/ $75 \mathrm{mmHg}$ increases one's risk of cardiovascular disease and mortality, it lowered the standard adult cutoff value of hypertension. In 2016, the European Society of Hypertension (ESH) guidelines suggested that a normal BP was less than the 90th percentile for persons aged 1-16 years and less than 130/85 mmHg for persons aged over 16 years. ${ }^{2)}$ In 2017, the American Academy of Pediatrics (AAP) guidelines changed the standard of an elevated pediatric BP to that above the 90th-95th percentiles by height, sex, and age for persons aged 1-13 years and above $120 / 80 \mathrm{mmHg}$ for persons over 13 years of age in line with the decrease in adult standard ${ }^{3-6}$ (Table 1). If BP measured by the oscillometric device is above the 90th percentile, auscultatory $\mathrm{BP}$ should be measured repeatedly, and if the average of $\mathrm{BP}$ is also higher than the 90th percentile, it is considered elevated; in contrast, if it is less than the 90th percentile, it is considered normal and monitored with an oscillometric device. ${ }^{5)}$ The ESH recommended the confirmation of hypertension using an auscultatory device since oscillometric BP measurements are usually higher than auscultatory BP measurements. ${ }^{2)}$ Considering these 2 guidelines, elevated BP is diagnosed using an auscultatory device in pediatric patients.

\section{Definition of neonatal hypertension}

The measurement of $\mathrm{BP}$ in newborns is important but difficult. Newborns, especially preterm infants, undergo rapid physiological changes before and after birth, but possible rapid changes in blood flow and BP make it difficult to classify measured BP. Neonatal hypertension is identified when the systolic BP measured 3 times is higher than the 95th percentile in the BP classification by gestational age, birth weight, and sex. ${ }^{6,7)}$ The evaluation of hypotension in newborns is also important. Hypotension is identified when the systolic BP is less than the 5 th-10th percentiles by gestational and postnatal age. ${ }^{7)}$ In extremely low birth weight infants, information on a normal $\mathrm{BP}$ is insufficient; therefore, mean arterial $\mathrm{BP}$, which is lower than the newborn's gestational age in weeks, is clinically used as a treatment criterion. ${ }^{7}$ In neonates, especially in cases of arterial catheterization through the umbilical artery, intraarterial BP through the catheter is the gold standard method for neonatal BP measurements. ${ }^{6,8)}$ Neonatal movement, feeding, catheter position, and appropriate catheter size for the vessel

Table 1. Definition of hypertension ${ }^{17,41,42)}$

\section{Neonates}

\begin{tabular}{|c|c|c|}
\hline Definition of HTN & $\geq 95$ th percentile for gestational age, birthweight, and sex on 3 separate occasions & \\
\hline Significant HTN & 95th-99th percentile for age and sex & \\
\hline \multirow[t]{3}{*}{ Severe HTN } & $\geq 99$ th percentile for age and sex & \\
\hline & \multicolumn{2}{|l|}{ Children } \\
\hline & $1-13$ Years & $\geq 13$ Years \\
\hline Normal BP & $<90$ th percentile & $<120 /<80 \mathrm{mmHg}$ \\
\hline Elevated BP & $\geq 90$ th percentile to $<95$ th percentile or $120 / 80 \mathrm{mmHg}$ to $<95$ th percentile (whatever is lower) & $120 /<80$ to $129 /<80 \mathrm{mmHg}$ \\
\hline Stage $1 \mathrm{HTN}$ & $\geq 95$ th percentile to $<95$ th percentile $+12 \mathrm{mmHg}$, or $130 / 80$ to $139 / 89 \mathrm{mmHg}$ (whatever is lower) & $130 / 80$ to $139 / 89 \mathrm{mmHg}$ \\
\hline Stage 2 HTN & 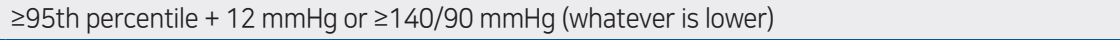 & $\geq 140 / 90 \mathrm{mmHg}$ \\
\hline
\end{tabular}

$\mathrm{BP}$, blood pressure; HTN, hypertension. 
may change intra-arterial BP; therefore, caution is required in judgment.9) However, if catheter insertion is not indicated, methods other than intra-arterial BP monitoring, which is highly invasive, should be considered. Oscillometric devices are used in newborns, as their Korotkoff sounds are almost inaudible using auscultatory devices.

\section{What are the auscultatory methods?}

Blood circulation in the body is caused by the continuous pumping action of the heart, which creates a pulsating flow when it drains blood from the left ventricle to the aorta. The systolic $\mathrm{BP}(\mathrm{SBP})$ is defined as the maximum pressure exerted against the inner walls of the blood vessels when the left ventricle contracts, while the diastolic BP (DBP) is defined as the force exerted during relaxation and dilation of the heart. ${ }^{10)}$ A noninvasive auscultatory method that easily measures arterial BP using a stethoscope and the bladder of the BP cuff was developed about 110 years ago by Nikolai Korotkoff. ${ }^{11)}$ Despite efforts to establish a superior BP measurement method, the auscultation method by Korotkoff remains the gold standard. Initially, Korotkoff described 4 sound phases, to which a fifth phase was added later. The pressure at the first audible sound during the slow loosening of the inflated cuff (Korotkoff phase I) was recorded as the SBP, while the pressure at the moment all sounds (Korotkoff phase V) disappear was recorded as the DBP. A sphygmomanometer and stethoscope are required to measure arterial BP using Korotkoff's auscultatory method.

A sphygmomanometer is a device attached to an inflatable bladder (cuff) through a rubber tube and consists of a pressure gauge or mercury column. The auscultation method using an MS is the gold standard for recording BP. An MS usually does not require calibration since the density of mercury cannot be changed, although periodic maintenance is necessary, such as filter cleaning. ${ }^{12)}$ Due to environmental and personal health concerns, international efforts have been made to discard healthcare sources of mercury in the thermometer and sphygmomanometer and replace them with less toxic alternatives. ${ }^{13,14)}$ Therefore, the clinical standard of auscultatory methods using mercury columns has given way to oscillometric devices in recent adult guidelines. ${ }^{15)}$ However, the MS is considered the standard device for BP measurement in epidemiological surveys in adults. ${ }^{16)}$ Moreover, in children, it should be noted that the BP table for the diagnosis of hypertension was made based on values using an MS. ${ }^{2,17)}$ There is a need for a method that can replace MS for measuring BP and diagnosing hypertension in children and adolescents.

\section{Aneroid sphygmomanometer}

The aneroid sphygmomanometer is a device in which a mercury pressure gauge is replaced by a set of bellows and mechanical springs. ${ }^{18)}$ However, unlike with MS, the aneroid sphygmomanometer may involve intermanufacturer differences in accuracy. Studies of its accuracy in clinical settings have reported varied results, with the frequency of defective devices varying from zero to more than $35 \%{ }^{18-20)}$ Another major concern of aneroid sphygmomanometers is the issue of maintenance and recalibration, which is not needed for the MS. This device should be regularly (no more than every 6 months) calibrated for accuracy using dynamic calibration methods as recommended in validation protocols. ${ }^{21)}$ A simple way to check the calibration is to verify that the needle is not pointed to $0 \mathrm{mmHg}$ when the manometer valve is open. ${ }^{12)}$

\section{1) Advantages}

Auscultatory BP measurement directly measures SBP and DBP through Korotkoff sounds. It is inexpensive and portable and does not require electricity, making it the gold standard for pediatric BP measurement and hypertension diagnosis. ${ }^{3-5)}$

\section{2) Disadvantages}

A common disadvantage of the auscultatory method is the requirement for an experienced examiner to perform the measurement. Also, mismeasurements due to examiner error are possible (Korotkoff sounds are difficult to hear, especially in young children), resulting in the underestimation of SBP or confusion of Korotkoff phases IV and $\mathrm{V}^{10)}$ As mentioned above, auscultating the Korotkoff sounds using small vessels of neonates is difficult, and pressing the stethoscope down on the arm causes bias since it increases the diastolic pressure. Therefore, use of the auscultatory method for newborns is not recommended. ${ }^{9)}$

\section{Hybrid sphygmomanometer}

Hybrid sphygmomanometers are another type of aneroid sphygmomanometer that combines electronic and auscultatory devices. The measurement of $\mathrm{BP}$ with a stethoscope is the same as that of an MS. ${ }^{18,22)}$ The Accoson Greenlight 300, a hybrid sphygmomanometer, is the first nonautomated and mercuryfree device that meets the international protocol for adults with guaranteed accuracy. It can fulfill the desire expressed by a consensus of experts representing the AHA. ${ }^{23,24)}$ Thereafter, it became known that devices such as the A\&D UM101 hybrid device (A\&D Co., Ltd., Toshima Ku, Tokyo, Japan), Pic Indolor Professional hybrid device (Pic Indolor Professional, Artsana Co., Milan, Italy), and Nissei DM3000 (Nissei Japan Precision Instruments, Gunma, Japan) could replace the MS in the validation study of the ESH international protocol 2010. ${ }^{25}$ Hybrid devices also have advantages of improving auscultatory BP measurements as they have a self-zeroing feature; thus, they can obviate the surveillance protocol that is required to ensure aneroid BP measurement device accuracy. ${ }^{24)}$

\section{1) Advantages}

The auscultatory method remains the gold standard for diagnosing hypertension in pediatric patients. Hybrid devices have an electronic pressure gauge but do not use a specific manufacturer's algorithm. The cuff pressure is accurately displayed 
on a digital light-emitting diode to eliminate terminal digit preference by the measurer. ${ }^{18)}$

\section{What are the oscillometric devices?}

Oscillometric devices have the advantage of eliminating human observer errors since they all provide a digital readout of the BP. Unlike the auscultatory method, oscillometry calculates a person's SBP and DBP, and each manufacturer of oscillometric devices uses its own algorithm for the calculation by examining the reflected oscillating pulse wave that is generated inside the deflating bladder. ${ }^{10,12)}$ A typical oscillometric device automatically inflates the cuff and then gradually deflates it to a pressure below the diastolic pressure. The oscillations begin above the systolic pressure and continue below the diastolic pressure so the SBP and DBP can only be estimated indirectly according to some empirically derived algorithms. Even a child's small movements can affect oscillometric pulses, which are essential to determining an accurate BP; therefore, it is challenging to measure BP in children who cry, shiver, or move.

The empirical nature of oscillometry requires validation of each model's accuracy. ${ }^{26)}$ Although many devices are marketed by different manufacturers, less than $15 \%$ pass validation studies. This issue is crucial in children, in whom few devices have been successfully validated using an established protocol. ${ }^{27)}$ Recent studies described models that have been validated in children, and we can read a device's user manual to see whether validation was performed and what protocol was used. ${ }^{27)}$ Oscillometric devices are convenient because their measurements are fully automated, they do not require training, and they have good interrater reliability. However, their accuracy in children remains uncertain. Each method mentioned above has advantages and disadvantages (Table 2).

\section{Ambulatory BP monitoring}

Ambulatory BP monitoring (ABPM) is a method of repeatedly measuring BP several times over a certain period (usually 24 hours). ABPM uses the oscillometric and auscultatory methods, but the former is mainly used because of the disadvantages of the latter, specifically measurement difficulties and the absence of a normal reference value. The BP cuff is wrapped around the arm, a small monitor is connected, and the BP is repeatedly measured every 15-30 minutes while the device is connected to the body. The measured BP is stored, and when the test is completed, the data are downloaded to the computer and analyzed. ${ }^{28,29)}$ The interpretation of the results follows AHA guidelines. Through this method, the mean value and day/nighttime ambulatory BP information of BP are provided to enable the identification of hidden variations of hypertension. ABPM can confirm an office

Table 2. The advantages and disadvantages of each method ${ }^{10}$

\begin{tabular}{|c|c|c|c|}
\hline & Aneroid auscultation & Hybrid auscultation & Oscillometry \\
\hline $\begin{array}{l}\text { Method of } \\
\text { BP estimation }\end{array}$ & $\begin{array}{l}\text { Detection of Korotkoff sounds through a } \\
\text { stethoscope for auscultation }\end{array}$ & $\begin{array}{l}\text { Combination of features of electronic and } \\
\text { auscultatory devices (auscultating Koro- } \\
\text { tkoff sounds and indicating cuff pressure } \\
\text { on digital light-emitting diode readout) }\end{array}$ & $\begin{array}{l}\text { Detection of arterial flow (oscillometry) in } \\
\text { which pulses sensed through the cuff are } \\
\text { filtered, amplified, processed, and applied } \\
\text { to an algorithm to estimate systolic and } \\
\text { diastolic BP }\end{array}$ \\
\hline Advantages & $\begin{array}{l}\text { - Direct estimation of SBP/DBP (more } \\
\text { accurate) } \\
\text { - Inexpensive and portable } \\
\text { - Dose not require electricity }\end{array}$ & $\begin{array}{l}\text { - Same as aneroid } \\
\text { - No need for regular recalibration } \\
\text { - Cuff pressure exactly indicated as a digital } \\
\text { light-emitting diode value, resulting in } \\
\text { elimination of the terminal digit prefer- } \\
\text { ence }\end{array}$ & $\begin{array}{l}\text { - Portable } \\
\text { - Easy to use; saves time and clinical } \\
\text { resources (less expertise and training } \\
\text { required when used in the absence of a } \\
\text { healthcare provider) } \\
\text { - Fewer observer errors; minimal observer } \\
\text { bias or terminal digit preference } \\
\text { - Good for screening, home use }\end{array}$ \\
\hline Disadvantages & $\begin{array}{l}\text { - Risk of noise interference } \\
\text { - Expertise and retraining required to avoid } \\
\text { observer error; (1) requires manual dex- } \\
\text { terity to ensure a proper cuff deflation rate, } \\
\text { (2) requires excellent hearing and vision } \\
\text { - Risk of observer bias and terminal digit } \\
\text { preference } \\
\text { - Requires regular calibration (at least every } 6 \\
\text { months); device can lose calibration } \\
\text { (become inaccurate) when jostled or } \\
\text { bumped, leading to false readings }\end{array}$ & $\begin{array}{l}\text { - Same as aneroid except for bias of ter- } \\
\text { minal digit preference } \\
\text { - Very few devices available to date }\end{array}$ & $\begin{array}{l}\text { - Requires access to a continuous power } \\
\text { source (electricity or battery) } \\
\text { - Requires validation by a standard protocol } \\
\text { (some are only for adults); some are } \\
\text { inaccurate; manufacturer variation due to } \\
\text { proprietary algorithm for estimation } \\
\text { - Device cost and longevity } \\
\text { - Must be replaced periodically because of } \\
\text { mechanical failure } \\
\text { - Many are not suitable for patients with } \\
\text { atrial fibrillation, decreased arterial com- } \\
\text { pliance, motion, or crying }\end{array}$ \\
\hline Pediatric area & \multicolumn{2}{|c|}{$\begin{array}{l}\text { - To date, it is the gold standard for hypertension diagnosis } \\
\text { - In infant/young children, SBP may be underestimated due to poor hearing of Korotkoff } \\
\text { sounds, or DBP may be incorrectly measured by confusion of Korotkoff phases IV and V }\end{array}$} & $\begin{array}{l}\text { - It can be applied when auscultation is } \\
\text { difficult, such as in small children or } \\
\text { newborns } \\
\text { - When applied to 24-hour ABPM, it can } \\
\text { detect masked hypertension and help } \\
\text { diagnose hypertension in patients with a } \\
\text { high auscultatory BP }\end{array}$ \\
\hline
\end{tabular}

BP, blood pressure; DBP, diastolic BP; SBP, systolic BP; AMBP, ambulatory blood pressure monitoring. 
elevated BP since it excludes white-coat hypertension or detects masked hypertension; moreover, it can better predict future BP after adulthood and is known to be particularly related to endorgan changes (ex, left ventricular hypertrophy). Therefore, the AAP guidelines recommend that children and adolescents with a high office BP undergo ABPM to confirm hypertension and that clinicians consider performing annual screenings of high-risk groups such as those with chronic kidney disease and diabetes. ${ }^{5)}$ This method is technically difficult in children under 5 years of age. ${ }^{28)}$

\section{1) Advantages}

As this method does not require measurer expertise, it is easy to access, removes measurer bias, and eliminates whitecoat hypertension because it can be measured by the patient alone. ${ }^{18,28,30)}$ Continuous BP measurements can be performed in a short amount of time. The advantage of electronic monitors is that they are less likely to cause inaccuracies in mechanical devices, such as mercury or aneroid devices, since they eliminate terminal digit preference by the measurer. It is good to use as a method of hypertension screening tests, such as school checkups. As mentioned above, it is difficult to hear the Korotkoff sounds using auscultation in neonates or infants, so the oscillometric method is applied instead.

\section{2) Disadvantages}

This is an indirect BP measurement, as BP is measured by calculating SBP and DBP from the mean arterial BP measured using each manufacturer's algorithm. In addition, the algorithm varies for among companies, so BP may vary accordingly. In addition, the measurement value may vary among patients with different underlying diseases, so it is difficult to trust the measurement value in cases of arrhythmia, and the accuracy of the result is theoretically poor for elderly, diabetic, and dialysis patients with poor arterial compliance. ${ }^{28,29)}$

\section{Comparisons of mercury and other devices}

\section{Mercury vs. aneroid sphygmomanometer}

Only a few studies have compared the accuracy of mercury versus aneroid sphygmomanometers in children. A large study published in 2012 demonstrated that mean mercury and aneroid systolic and diastolic BP were highly correlated. There was no significant difference in mean SBP, but a lower mean DBP (by $-1.53 \pm 5.06 \mathrm{mmHg}$ ) was seen in the aneroid device group. Although a small discrepancy in DBP measurements exists between aneroid and mercury devices, this variation is unlikely to be clinically significant, suggesting that either device could be used in research or clinical settings. ${ }^{31)}$ Another comparison study using data of 727 individuals (127 of whom were 8-17 years old) in the U.S. National Health and Nutrition Examination Surveys showed that the mean readings were not significantly different between the 2 methods except for SBP in patients aged 8-17 years (mean difference, $1.10 \mathrm{mmHg}$ ) ${ }^{16}$ ) In a recent systematic review article, only 3 studies compared aneroid and MS. ${ }^{32)}$ In an additional study on adults in 2015, the BP measured using mercury, aneroid, and digital devices in 108 subjects were analyzed by paired t test. The average SBP was $124.57 \mathrm{mmHg}$ for digital devices, $124.31 \mathrm{mmHg}$ for aneroid, and $125.01 \mathrm{mmHg}$ for mercury, and there was no significant difference in mercuryaneroid, mercury-digital, and aneroid-digital measurements, so it was argued that a digital device or an aneroid device could replace a mercury device. ${ }^{33)}$

\section{Mercury vs. oscillometric device}

A recent meta-analysis (random-effects model) analyzed 34 studies comparing oscillometric devices with MS performed before July 2015.22) The oscillometric devices showed higher SBP than MS, with a pooled effect of about $2.53 \mathrm{mmHg}(95 \%$ confidence interval [Cl], $0.57-4.5 ; P<0.05)$. In the studies that passed validation, the pooled effect decreased to $1.75 \mathrm{mmHg}$ $(95 \% \mathrm{Cl}, 0.61-2.81 ; \mathrm{n}=12)$. There was no significant difference in DBP, which is defined as Korotkoff phase V. However, in some studies that defined DBP as Korotkoff phase IV by auscultation, oscillometric devices underestimated DBP. The limitation of this study is the large interstudy heterogeneity. Studies in clinical situations other than school-based studies found no differences, although these studies comprised only a small proportion of all studies. ${ }^{32)}$ A study comparing a mercury device and an oscillometric device in 77 obese pediatric patients aged 5-17 years was published in 2017. The mercury device showed significantly lower SBP and DBP values than the oscillometric device (Omron HEM 725 CIC, OMRON Healthcare, Kyoto, Japan) at $104.0 \pm 9.6 \mathrm{mmHg} / 61.1 \pm 9.5 \mathrm{mmHg}$ for the mercury device and $107.8 \pm 14.5 \mathrm{mmHg} / 71.4 \pm 15.8 \mathrm{mmHg}$ for the electronic device. ${ }^{34)}$ Another meta-analysis published in 2019 compared measurement methods among patients older than 15 years of age in 24 papers and found that the oscillometric device had a lower SBP (mean difference, $-1.75 \mathrm{mmHg}$; 95\% CI, -3.05 to $-0.45 \mathrm{mmHg} ; I^{2}=91.0 \%$ ) and DBP (mean difference, $-1.20 \mathrm{mmHg}$; $95 \% \mathrm{CI},-2.16$ to $-0.24 \mathrm{mmHg}$; $\left.I^{2}=95.0 \%\right),{ }^{35)}$ showing contradictory results with those of the previous study. A sub-group analysis revealed differences among manufacturing companies. The BpTRU monitor (VSM MedTec Ltd., Vancouver, Canada) showed lower results than mercury devices (SBP, $-4.08 \mathrm{mmHg}$; DBP, $-2.5 \mathrm{mmHg}$ ), and there was no significant difference with the OMRON BP mercury device (OMRON Healthcare), but the results differed among devices. However, this study has a limitation in that its heterogeneity was exceedingly high. It is important to establish a validation protocol for the algorithm because the studies published to date reported conflicting conclusions and differences among manufacturers. In addition, more research is needed to determine whether oscillometric devices can replace mercury devices in pediatric patients. 


\section{Next-generation BP measurements in the mobile era}

Recently, as the tools for BP measurement have diversified and the mobile market has evolved, a method using a mobile application for BP measurement has emerged. Instead of wrapping the bladder cuff around the arm and pumping air into it, a user simply places a finger on the smartphone to measure BP and checks the results through the mobile application. When the patient puts a finger on the smartphone camera sensor, the sensor emits a 940-nm light-emitting diode, the light of which reaches the photodetector after passing through the finger. ${ }^{30)}$ This photoplethysmography (PPG) signal detects changes in blood flow corresponding to the patient's natural pulsation, and SBP and DBP can be measured according to the algorithm of each application. In 2020, traditional BP using an arterial catheter and BP using a smartphone were simultaneously measured and analyzed in 50 adults to determine the accuracy of "BP measured by applying the PPG signal obtained from the smartphone to the dedicated app (OptiBP)". ${ }^{36}$ ) The difference between the 2 values showed only the difference of SBP $-0.7 \pm 7.7 \mathrm{mmHg}$, DBP $-0.4 \pm 4.5 \mathrm{mmHg}$, and mean BP $-0.6 \pm 5.2 \mathrm{mmHg}$, confirming the accuracy of mobile application-based BP. Another study compared the BP of senior nursing students (aged over 18 years) using a traditional automated BP cuff versus that using PPG signals in Apple iOS health applications. The article reported that DBP was significantly overestimated by the mobile application at SBP $122.94 \mathrm{mmHg} / \mathrm{DBP} 76.62 \mathrm{mmHg}$ in the traditional method and SBP $125.72 \mathrm{mmHg} / \mathrm{DBP} 81.86 \mathrm{mmHg}$ in the mobile method. ${ }^{37}$ No studies have examined smartphone-based PPG using BP measurements in children. However, in a study comparing $\mathrm{BP}$ with standard $\mathrm{ABPM}$ using pulse transit time, a cuffless BP measurement method, the mean SBP and DBP of 30 patients aged 10-18 years with primary hypertension or a high normal BP were evaluated. As a result, the cuffless device showed a significantly higher BP (standard vs. cuffless device: SBP $123.47 \pm 14.91 \mathrm{mmHg}$ and $127.48 \pm 15.98 \mathrm{mmHg}, P<0.001$; DBP $66.88 \pm 11.86 \mathrm{mmHg}$ and $68.52 \pm 12.36 \mathrm{mmHg}, P<$ 0.001). ${ }^{38)}$ More research in pediatric populations is needed, and a certified validation protocol for each manufacturer's device and algorithm should be developed. The World Health Organization technical specification for an automated noninvasive BP measuring device with a cuff showed concern that $\mathrm{BP}$ measurements using smartphone application are in the early stages of research and development and may lead to wrong clinical decision due to the absence of validation protocols and inaccuracy of measurements and expressed the expectation that it improves hypertension diagnostics by increasing interest and ease of BP measurement. ${ }^{30)}$ It is currently in the early stage of research, and we expect that the subject will be expanded to children and adolescents in the future. If the problem of low accuracy is improved, it will be possible to improve the quality of medical care and reduce medical costs for vulnerable populations.

\section{Which device will replace the MS in children?}

A recent study of Korean adults compared the accuracy and errors of BP measured by 2 types of non-MS devices in an epidemiologic survey. ${ }^{39)}$ The researchers alternatively used an MS and 2 electronic devices and calculated the BP measurement differences, defined as BP measured by MS minus BP obtained by 2 electronic devices. The concordance correlation coefficients were not significantly different for SBP (mean differences: -0.52 and $-0.62 \mathrm{mmHg}, P=0.76$; concordance correlation coefficients: 0.97 and 0.94 ), but the concordance correlation coefficient for DBP were significantly different among manufacturers (mean differences: -0.78 and $6.23 \mathrm{mmHg}, P<0.01$; concordance correlation coefficients: 0.95 and 0.76 ). ${ }^{39)}$ In 2018, a similarly designed study of the Korean National Health and Nutrition Examination Survey Program was conducted in children over 10 years of age to determine which BP measurement device is most suitable for replacing MS in future surveys. A direct comparison among the 3 devices under well-controlled BP measurement protocols suggested significant differences among manufacturers in children and adolescents as seen in adults, especially for DBP (unpublished data). Two recent guidelines for hypertension in children recommended that oscillometric devices may be used for BP screening in children, and if they detect hypertension, the values must be confirmed using the auscultatory method. They emphasize that at least $3 \mathrm{BP}$ measurements obtained at rest by manual auscultation are essential when diagnosing a child or adolescent with hypertension., ${ }^{2,17,40)}$ ABPM may also help diagnose hypertension in cases of elevated manual auscultatory BP values. ${ }^{3,5)}$

In conclusion, although oscillometric devices have become widely used in children, they have not yet completely replaced MS or other auscultatory methods, especially for confirming hypertension in children. The aneroid or hydrid auscultatory device can be a good alternative to MS, and hybrid devices have the advantage that they usually do not require regular calibration of aneroid devices. There is a need for further discussion about a BP measurement device that can replace mercury, and it can be said that the mobile application-based BP measurement has very high development potential because of its convenience and accessibility, even though it is still in the early stages of research.

\section{Footnotes}

Conflicts of interest: No potential conflict of interest relevant to this article was reported.

Acknowledgments: I acknowledge the Korean Working Group of Pediatric Hypertension members dedicated to the research and treatment of hypertension in children.

ORCID:

Seong Heon Kimi @ https://orcid.org/0000-0001-8003-3010 


\section{References}

1. Eliasdottir SB, Steinthorsdottir SD, Indridason OS, Palsson R, Edvardsson VO. Comparison of aneroid and oscillometric blood pressure measurements in children. J Clin Hypertens (Greenwich) 2013;15:776-83.

2. Lurbe E, Agabiti-Rosei E, Cruickshank JK, Dominiczak A, Erdine S, Hirth A, et al. 2016 European Society of Hypertension guidelines for the management of high blood pressure in children and adolescents. J Hypertens 2016;34:1887-920.

3. Ashraf M, Irshad M, Parry NA. Pediatric hypertension: an updated review. Clin Hypertens 2020;26:22.

4. Benenson I, Waldron FA, Porter S. Pediatric hypertension: a guideline update. Nurse Pract 2020;45:16-23.

5. Weaver DJ Jr. Pediatric hypertension: review of updated guidelines. Pediatr Rev 2019;40:354-8.

6. Giri P, Roth P. Neonatal hypertension. Pediatr Rev 2020;41:307-11.

7. Park HK. Optimal blood pressure in preterm infants. Neonatal Med 2014;21:99-105.

8. Dionne JM, Bremner SA, Baygani SK, Batton B, Ergenekon E, BhattMehta V, et al. Method of blood pressure measurement in neonates and infants: a systematic review and analysis. J Pediatr 2020;221:23-31.e5.

9. Moss AJ. Blood pressure in infants children and adolescents. West J Med 1981;134:296-314.

10. James GD, Gerber LM. Measuring arterial blood pressure in humans: Auscultatory and automatic measurement techniques for human biological field studies. Am J Hum Biol 2018;30(1). https://doi.org/10.1002/ ajhb.23063. [Epub].

11. Lewis WH. The evolution of clinical sphygmomanometry. Bull NYAcad Med 1941;17:871-81.

12. Flynn J, Ingelfinger JR, Portman RJ. Pediatric hypertension. Totowa (NJ): Humana Press, 2013:131-40.

13. Messelbeck J, Sutherland L. Applying environmental product design to biomedical products research. Environ Health Perspect 2000;108 Suppl 6(Suppl 6):997-1002.

14. Buchanan S, Orris P, Karliner J. Alternatives to the mercury sphygmomanometer. J Public Health Policy 2011;32:107-20.

15. Whelton PK, Carey RM, Aronow WS, Casey DE Jr, Collins KJ, Dennison Himmelfarb C, et al. 2017 ACC/AHA/AAPA/ABC/ACPM/AGS/APhA/ ASH/ASPC/NMA/PCNA guideline for the prevention, detection, evaluation, and management of high blood pressure in adults: a report of the American College of Cardiology/American Heart Association Task Force on Clinical Practice Guidelines. Hypertension 2018;71:e13-115.

16. Ostchega Y, Prineas RJ, Nwankwo T, Zipf G. Assessing blood pressure accuracy of an aneroid sphygmomanometer in a national survey environment. Am J Hypertens 2011;24:322-7.

17. Flynn JT, Kaelber DC, Baker-Smith CM, Blowey D, Carroll AE, Daniels SR, et al. Clinical practice guideline for screening and management of high blood pressure in children and adolescents. Pediatrics 2017;140: e20171904.

18. Pickering TG. What will replace the mercury sphygmomanometer? Blood Press Monit 2003;8:23-5.

19. Bailey RH, Knaus VL, Bauer JH. Aneroid sphygmomanometers. An assessment of accuracy at a university hospital and clinics. Arch Intern Med 1991;151:1409-12.

20. Burke MJ, Towers HM, O'Malley K, Fitzgerald DJ, O'Brien ET. Sphygmomanometers in hospital and family practice: problems and recommendations. Br Med J (Clin Res Ed) 1982;285:469-71.

21. Waugh JJ, Gupta M, Rushbrook J, Halligan A, Shennan AH. Hidden errors of aneroid sphygmomanometers. Blood Press Monit 2002;7:30912.

22. Pickering T. The case for a hybrid sphygmomanometer. Blood Press Monit 2001;6:177-9.

23. Jones DW, Frohlich ED, Grim CM, Grim CE, Taubert KA. Mercury sphygmomanometers should not be abandoned: an advisory statement from the Council for High Blood Pressure Research, American Heart Association. Hypertension 2001;37:185-6.

24. Graves JW, Tibor M, Murtagh B, Klein L, Sheps SG. The Accoson Greenlight 300, the first non-automated mercury-free blood pressure measurement device to pass the International Protocol for blood pressure measuring devices in adults. Blood Press Monit 2004;9:13-7.

25. Stergiou GS, Karpettas N, Kollias A, Destounis A, Tzamouranis D. A perfect replacement for the mercury sphygmomanometer: the case of the hybrid blood pressure monitor. J Hum Hypertens 2012;26:220-7.

26. Amoore JN. Oscillometric sphygmomanometers: a critical appraisal of current technology. Blood Press Monit 2012;17:80-8.

27. Stergiou GS, Boubouchairopoulou N, Kollias A. Accuracy of automated blood pressure measurement in children: evidence, issues, and perspectives. Hypertension 2017;69:1000-6.

28. Gillman MW, Cook NR. Blood pressure measurement in childhood epidemiological studies. Circulation 1995;92:1049-57.

29. Ogedegbe G, Pickering T. Principles and techniques of blood pressure measurement. Cardiol Clin 2010;28:571-86.

30. World Health Organization. WHO technical specifications for automated noninvasive blood pressure measuring devices with cuff. Geneva (Switzerland): World Health Organization, 2020.

31. Shah AS, Dolan LM, D'Agostino RB Jr, Standiford D, Davis C, Testaverde $\mathrm{L}$, et al. Comparison of mercury and aneroid blood pressure measurements in youth. Pediatrics 2012;129:e1205-10.

32. Duncombe SL, Voss C, Harris KC. Oscillometric and auscultatory blood pressure measurement methods in children: a systematic review and meta-analysis. J Hypertens 2017;35:213-24.

33. Hamied LIA, Sofiatin Y, Rakhmilla LE, Putripratama AA, Roesli RMA. Comparison of mercury, aneroid and digital sphygmomanometer in community setting. J Hypertension 2015;33:e33-4.

34. Fonseca-Reyes S, Romero-Velarde E, Torres-Gudiño E, Illescas-Zarate D, Forsyth-MacQuarrie AM. Comparison of auscultatory and oscillometric $\mathrm{BP}$ measurements in children with obesity and their effect on the diagnosis of arterial hypertension. Arch Cardiol Mex 2018;88:16-24.

35. Park SH, Park YS. Can an automatic oscillometric device replace a mercury sphygmomanometer on blood pressure measurement? A systematic review and meta-analysis. Blood Press Monit 2019;24:265-76.

36. Schoettker P, Degott J, Hofmann G, Proença M, Bonnier G, Lemkaddem A, et al. Blood pressure measurements with the OptiBP smartphone app validated against reference auscultatory measurements. Sci Rep 2020; 10:17827.

37. Bove LA, Carroll R. Comparing traditional blood pressure with a mobile health application [Internet]. Chicago (IL): Healthcare Information and Management Systems Society; 2020 [cited 2021 Aug 26]. Available from: https://www.himss.org/resources/comparing-traditional-blood-pre ssuremobile-health-application.

38. Zachwieja J, Neyman-Bartkowiak A, Rabiega A, Wojciechowska M, Barabasz M, Musielak A, et al. Comparison of cuff-based and cuffless continuous blood pressure measurements in children and adolescents. Clin Exp Hypertens 2020;42:512-8.

39. Choi S, Kim YM, Shin J, Lim YH, Choi SY, Choi BY, et al. Comparison of the accuracy and errors of blood pressure measured by 2 types of nonmercury sphygmomanometers in an epidemiological survey. Medicine (Baltimore) 2018;97:e10851.

40. Brady TM, Stefani-Glücksberg A, Simonetti GD. Management of high blood pressure in children: similarities and differences between US and European guidelines. Pediatr Nephrol 2019;34:405-12.

41. Dionne JM, Abitbol CL, Flynn JT. Hypertension in infancy: diagnosis, management and outcome. Pediatr Nephrol 2012;27:17-32.

42. Report of the Second Task Force on Blood Pressure Control in Children-1987. Task Force on Blood Pressure Control in Children. National Heart, Lung, and Blood Institute, Bethesda, Maryland. Pediatrics 1987;79:1-25.

43. Batton B. Neonatal blood pressure standards: what is "normal"? Clin Perinatol 2020;47:469-85. 
How to cite this article: Lee $\mathrm{SH}$, Kim SH. Blood pressure measurements and hypertension in infants, children, and adolescents: from the postmercury to mobile devices. Clin Exp Pediatr 2022;65:73-80. https://doi.org/10.3345/cep.2021.00143 